\title{
Task Environments for the Dynamic Development of Behavior
}

\author{
Derek Harter and Robert Kozma \\ Department of Mathematical Sciences; University of Memphis \\ Memphis, TN 38152 USA \\ \{dharter, rkozma\}@memphis.edu \\ http://www.psyc.memphis.edu/\{ harterd, kozmar $\}$
}

\begin{abstract}
The development of complex, adaptive behavior in biological organisms represents vast improvement over current methods of learning for artificial autonomous systems. Dynamical and embodied models of cognition [1-13] are beginning to provide new insights into how the chaotic, non-linear dynamics of heterogeneous neural structures may self-organize in order to develop effective patterns of behavior. We are interested in creating models of ontogenetic development that capture some of the flexibility and power of biological systems. In this paper we present a testbed for the creation and testing of models of development. We present some results on standard neural networks in learning to perform this task and discuss future plans for developmental models in this environment.
\end{abstract}

\section{Introduction}

\subsection{Development and Non-linear Dynamics}

The development of behavior in biological organisms is primarily a self-organizing phenomenon. Organisms are born with a basic repertoire of motor skills and instinctive needs. These are often tied to simple action-loops 1], which provide a basic repertoire of simple pattern completion and instinctive behaviors that can begin to satisfy the intrinsic drives of the organism. As the organism develops both physically and behaviorally, however, these instinctive behavior patterns begin to be associated with more general sensory stimuli. The organism learns to recognize patterns in the environment that are important and useful affordances for beneficial behaviors [14]. Increasingly complex patterns of behavior are organized around the solutions that are discovered at earlier stages of development.

Thelen and Smith [1315] view development as a shifting ontogenetic landscape of attractor basins. As physical and behavioral patterns develop the landscape is continually reformed and reshaped. Each developed behavior opens up many possibilities for new more complex patterns of behavior, while closing off possibilities for others. Even relatively simple tasks can provide opportunities 
for the development of increasingly complex strategies in order to improve performance. For example in the simple task we present in the next section, humans develop higher level strategies for improving their performance.

Many theories of the development of behavior in biological organisms are beginning to view it in terms of a self-organizing dynamical system [13/9]8. The organization of patterns of behavior is viewed, in some sense, as the formation and evolution of attractor landscapes. Some research 12 5/4 4 6/7/10 also indicates that chaotic dynamics may play an essential part in the formation of perception and behavior in biological organisms.

\subsection{Category Formation through Aperiodic Chaotic Dynamics}

Following experimental evidence and theoretical modeling [5[10], categories are associated with localized wings of a complex, high-dimensional chaotic attractor. The attractor landscape is formed in a flexible way reflecting past experiences and continuously modified based on the actual information the system receives from the environment.

The system typically resides in a high-dimensional basal state. It can be kicked-off from this state in response to external factors or internal developments. As the result, the state of the system is switched to a low-dimensional wing, which might represent a previously learnt memory pattern or an elementary action. The system might reside in this wing or it may visit sequentially various wings, thus representing a possible behavioral pattern. In either case, the system moves back to the basal state upon completing the sensory inputinduced identification task or performing the desired action. The advantage of such a dynamical approach is the flexibility and robustness of the selection of the behavior and action as it is seen in biological systems. We attempt to implement this dynamical strategy to solve action selection tasks as outlined in this work.

\subsection{Task Environments for Testing Models of Development}

Biological organisms are capable of marvelously complex patterns of behavior in pursuit of the satisfaction of their endogenous drives. However, it is not always apparent how much of the complexity of their behavior is internally generated, and how much emerges from the interaction of simple response patterns within a complex task environment. It seems doubtful that true progress in understanding the properties of intelligent behavior can be made by studying disembodied, syntactic systems $[161718$ 18. Intelligent behavior, at least in biological organisms, seems built upon a foundation of fast and robust pattern recognition and completion, both of static and temporally extended, often vague and noisy patterns. This observation is suggestive of several features that may be necessary in the developmental processes of biological organisms.

Of course it is desirable to develop models of behavior in realistic and complex environments, but it is not always possible. The question then becomes: what are the features of real world environments that are necessary for the development of complex behavior in biological organisms. Can we begin to study 
the phenomenon of the dynamic development of behavior in such a way that our results are valid in more realistic, complex environments. The following are a few features of environments that seem critical in creating models of the development of behavior.

Real Time Constraints Invariably biological organisms face critical time pressures that constrain their behavior. It is not simply a matter of doing the right thing, biological organisms must do the right thing and do it in a timely manner. It is better to do something, even something not optimal, on time, than to be too late and become someone else's dinner.

Developmental processes occur within the real time interactions of the organism with the environment. A testbed for developmental processes should be able to support tasks with real time constraints on the behavior of the developing system. However, in order to begin creating models it is desirable to loosen the restrictions of real time constraints. Therefore the testbed should have configurations with and without real time constraints on behavior. The task should be similar enough in both configurations so that mechanisms developed without constraints can eventually be tried in the more demanding situation of tasks with real time constraints.

Multiple Sensory Modalities The task environment should support the simulation of multiple sensory modalities, so that associations can be formed between purely reactive (intrinsic) behaviors, and more complex senses and behavior. This type of learned association between events in disparate sensory modalities seems to be crucial to many types of category formation and learned behavior. For example, in classical conditioned learning, the co-occurrence of an auditory stimuli (bell) with onset of reward results in a conditioned response being developed [19202122].

Exploitable Environmental Regularities Even though an environment is complex, it still must posses statistically significant, exploitable regularities in order to be a viable, survivable niche. Such regularities may co-occur in spatial location and time, or be more spatially or temporally extended. A major part of developmental processes is discovering such regularities and learning to exploit them.

\section{Packing Task}

Towards the end of studying and creating models of development, we have begun work on creating appropriate tasks with the previous properties. We describe a packing task here which is one such environment, and some work on standard machine learning tools in this environment. 


\subsection{Description}

In the packing task, the behaving system is presented with a series of shapes, one shape at a time. In this packing task, which is a simplified form of the Tetris game [23], the system can be presented with one of 3 shapes as shown in figure 1. The goal of the task is to move and rotate a shape before allowing it to drop onto a playing field in such a way as to end up with as compact of a packing as possible. An example of a packing trial in progress can be found in figure 2

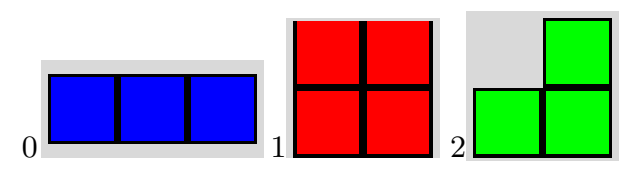

Fig. 1. The shapes used in the packing task.

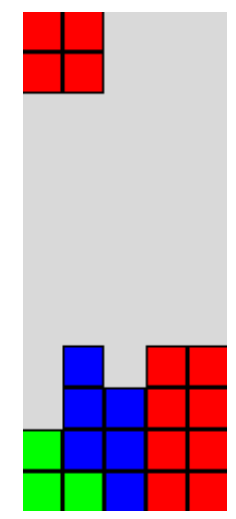

Fig. 2. An example packing task trial. Shapes enter from the top and must be positioned and rotated before they are dropped. Performance is evaluated by the height and the density of the packing of the shapes.

The behaving system does not know in advance what sequence of shapes it will be given. In our version of the packing task, the system is given random sequences of 10 shapes. The performance of the system on the packing task is evaluated by examining the density of their packing and by examining the total height of the resulting packing.

The system can produce two types of behavior. It must specify where to position (or move) the shape in the playing field, and how to rotate the shape. Once the system has specified the position and rotation of the shape, it is allowed to fall down onto the playing field. The shape settles into place and the next shape is presented to the behaving system to be positioned and rotated. 


\subsection{Encoding}

We now present an example of a standard neural network that learns to perform the simple packing task. The neural network needs to be given some sense of the current state of the environment. For the experiments performed here, two pieces of input were given to the network: the type of shape that has appeared, and a perception of the contours of the current playing field.

The encoding of the type of shape is relatively simple. In the packing task environment there are 3 different shape types. We used 2 bits to encode the type of shape. The shapes in figure 1 were given numbers (from left to right) of 0,1 and 2 and were encoded as 00,01 and 10 respectively.

The perception of the state of the playing field (the environment) is necessary in order to produce good behavior on where and how to position the shape before dropping it. In our reduced packing task, the playing field consisted of 5 columns. We sensed the height of each column currently in the environment, and encoded this for training and testing the networks. The lowest point in the playing field is used as a baseline and is encoded as having a height of 0 . All other heights are calculated from the baseline depth. We used 2 bits to encode the height of each column, and simply ignored perception of columns that were greater than 3 units above the baseline.

For example in figure 2, the leftmost column has the lowest depth in the playing field, and would be encoded with height 0 . The next column to the right has a height 2 units above the baseline. So from left to right, the height of the columns in figure 2 would be encoded as $0,2,1,2,2$. The type of shape shown in figure 2 about to be dropped is shape number 1 . As stated before we used 2 bits to encode the shape type, and 2 bits for each of the column heights, for a total of 12 bits of input. The situation shown in figure 2 would be encoded as:

Type Col1 Col2 Col3 Col4 Col5

$\begin{array}{llllllllllll}0 & 1 & 0 & 0 & 1 & 0 & 0 & 1 & 1 & 0 & 1 & 0\end{array}$

For the output of the system we developed the following encoding. We encoded the position to place the shape from the left edge in 3 bits. We need to be able to specify up to 5 units of displacement, thus we needed 3 bits to encode the 5 possibilities. The shapes can be rotated in increments of 90 degrees. Shape 2 (the L shape) can be rotated into 4 different distinct orientations. Therefore we also needed 2 bits to encode all possible specifications of rotation.

\subsection{Training}

We trained standard backpropogation networks using the encoding described above. For training data we had a human perform 50 packing trials, and we captured and encoded the input and the output of the behavior that the human produced when performing the packing task. We also captured a similar set of data for testing. We trained and tested the networks with many different configurations of number of hidden nodes and epochs trained. We then chose 
the best configurations in order to evaluate the performance of the networks on the packing task as discussed in the next section. The neural network performed best with 50 hidden nodes.

\subsection{Experiments}

We then used our packing task testbed in order to evaluate the performance of the networks on simulated packing trials. We gave the networks 100 random trials and measured their performance by calculating the packing density and height that they achieved. Packing height is simply a measure of the highest column of blocks in the playing field. Packing density is measured by looking at the ratio of the number of filled spaces in the packing to the total area of the packing. In figure 2 the packing has a height of 4 and a density of $17 / 20$ or 0.85 . The lower the height of the packing is the better the performance and similarly the denser the packing is the better the performance.

\subsection{Results}

A human learned the packing task and was asked to perform the task for 100 trials. Similarly the resulting neural networks were run on 100 trials of the packing task. Table 1 shows a comparison of the average performance on the 100 trials of the neural network and the human. Figure 3 shows a histogram of the performance of the human and the neural network rated by height and density.

Table 1. Comparison of average height and density performance measures on 100 simulated packing tasks

\begin{tabular}{lll}
\hline & Height & Density \\
\hline Human & 7.62 & 0.8748 \\
Neural Network & 8.18 & 0.8261 \\
\hline
\end{tabular}

Basic neural networks perform adequately on the packing task, but obviously are not quite as good at packing as humans, even for this simplified task domain. Humans, when performing this task, alter their strategies as the task progresses. Early on in a packing trial, a human is willing to leave open opportunities for particular shapes. People know intuitively that, even though they see shape types at random, they are likely to see the particular shape type needed if it is still early in the trial. However, as the trial progresses, strategies shift to those that will simply minimize the height of their packing.

This shift in strategies causes confusion for simple backpropogation networks. They see this as conflicting output patterns for the same input. Strategies that would possibly correct this deficiency for basic neural networks and other solutions will be discussed in the next section. 


\subsection{Discussion of Development in the Packing Task}

The development of differing strategies given the context of the problem is a prime example of the development of skills in biological organisms. People are not given explicit examples of appropriate shifts in strategies. They develop such strategies by interacting with the task environment, and guided by their previous experience with the constraints of the problem. They seem to quickly and intuitively embody the opportunities that situations afford for good behaviors, and how such opportunities change with the changing situation. In other words, they develop a set of skills and strategies for improving their performance on the problem simply through interaction and experience in the task domain.
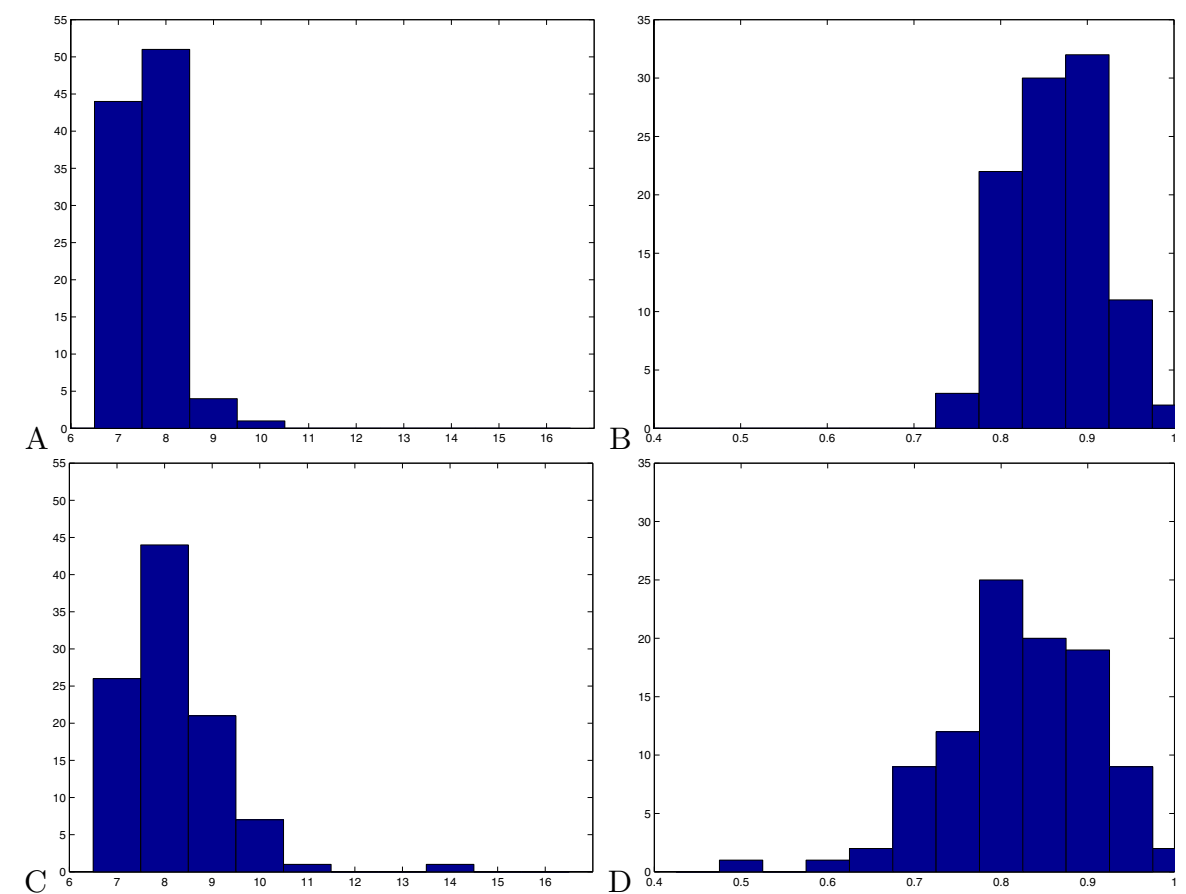

Fig. 3. Histograms of performance on 100 trials of the packing task. The top two figures (A and $\mathrm{B}$ ) show the performance of a human subject in the packing task, while the bottom two (C and D) display the performance of a neural network. In the left column we are measuring performance by the height of the packing. On the right we show performance by the density of the packing.

Even in our simple environment we see that people develop differing strategies for behavior based on the context of the progress of the trial. For example, 
humans first learn a basic set of good contours and correspondance with different shape types that provide for efficient packings. From this basic set of behavior, they begin to develop preferences for patterns that keep open future opportunities. For example, some contours will naturally accommodate more than one shape type, and are preferred over other patterns that limit good packing to a single shape type. Even further, people begin to develop higher level strategies at this point. For example, if it is early in the trial they wait for more optimal packings, but later on they simply try and minimize the height. The challenge in creating models of development is in capturing this ability to, not only softly assemble solutions through a repertoire of learned and innate skills, but to also develop new skill and effective higher level strategies for the problem domain.

\section{$3 \quad$ Future Directions}

The basic neural networks presented here are not quite capable of human level performance in the packing task. The primary reason for this deficiency is an inability to perceive the changes in circumstances that cause a shift in the behavior of the human trainers. We have no doubt that adding on more contextual input (such as the current height of the packing, or a count of the number of shapes packed so far) would improve the performance of the basic network, though it remains to be seen if it could equal human performance. Also, other methods such as recurrent, dynamical neural networks, or genetic algorithm optimizations, should be capable of bringing standard methods of machine learning up to human level performance on this simple task.

The point is not to equal human performance in this simplified domain, but to begin to create models that can develop behavior on their own in a cognitively plausible manner, and that display some of the flexibility of biological development. Most standard methods of machine learning should be able to competently handle the packing task environment in its simplified form but inevitably will break down as we add complexity and real time constraints to the task.

KIII is a dynamical memory device, which has been used successfully to solve difficult classification problems in vague, and noisy environments 10 . The KIII model incorporates several KII sets, which can be interpreted as units generating limit cycle oscillations in an autonomous regime. High-dimensional aperiodic and chaotic behavior does not emerge until the complete KIII system is formed. KIII has a multi-layer architecture with excitatory and inhibitory lateral, feed-forward, and feedback connections. KIII models can grasp the essence of the observed dynamic behavior in certain biological neural networks. It seems feasible to build a simplified version of KIII for the action selection task addressed in this work. We call it $3^{*} \mathrm{KII}$ model, as it consists of 3 mutually interconnected KII sets. Each KII set has a well-defined oscillation frequency. The complete $3^{*}$ KII model, however, may exhibit high-dimensional, aperiodic oscillations as the result of competing, incommensurate frequencies of the KII components. 
The advantage of $3^{*} \mathrm{KII}$ is that it allows a self-organized encoding of behavioral patterns into localized wings of a high-dimensional attractor. Therefore, we can obtain flexible and noise-resistant transitions among the states of the system, self-organized into a sequence of elementary actions of phase transitions. It is expected that defining a more challenging packing task with larger number and more complicated block patterns and also larger play field the application of dynamical encoding and action selection mechanism as $3^{*} \mathrm{KII}$ would prove to be beneficial. Also the emergence of self-organized action patterns would be imminent and complex behavioral patterns could be studied.

\section{Conclusion}

The development of behavior, even in a simplified environment such as the packing task, can shed light on the mechanisms of biological development and learning. Biological organisms are able to effectively develop increasingly complex skills and strategies simply by interacting with and solving problems in their environment. The dynamic, self-organization of behavior in biological organisms is a powerful model of learning that, if better understood, would provide great opportunities for improved artificial behaving and learning systems. Development of behavior in biological organisms can be viewed as a self-organizing dynamical system. Some research also indicates the importance of chaotic modes of organization in the development of behavior. We can begin to study models of development even in simplified ways as long as we are aware of the essential properties of the environments that are exploited by biological organisms during developmental process. Some of these properties include critical real time constraints, a rich sensory modality and environmental regularities and exploitable features.

\section{References}

1. Andy Clark. Being There: Putting Brain, Body, and World Together Again. The MIT Press, Cambridge, MA, 1997.

2. Gerald M. Edelman and Giulio Tononi. A Universe of Consciousness: How Matter Becomes Imagination. Basic Books, New York, NY, 2000.

3. Stanley P. Franklin. Artificial Minds. The MIT Press, Cambridge, MA, 1995.

4. Walter J. Freeman. Consciousness, intentionality and causality. In Núñez and Freeman [24], pages 143-172.

5. Walter J. Freeman. How Brains Make Up Their Minds. Weidenfeld \& Nicolson, London, 1999.

6. Walter J. Freeman and Robert Kozma. Local-global interactions and the role of mesoscopic (intermediate-range) elements in brain dynamics. Behavioral and Brain Sciences, 23(3):401, 2000.

7. Walter J. Freeman, Robert Kozma, and Paul J. Werbos. Biocomplexity: Adaptive behavior in complex stochastic dynamical systems. BioSystems, 2000.

8. Horst Hendriks-Jansen. Catching Ourselves in the Act: Situated Activity, Interactive Emergence, Evolution and Human Thought. The MIT Press, Cambridge, MA, 1996. 
9. J. A. Scott Kelso. Dynamic Patterns: The Self-organization of Brain and Behavior. The MIT Press, Cambridge, MA, 1995.

10. Robert Kozma and Walter J. Freeman. Chaotic resonance - methods and applications for robust classification of noisy and variable patterns. International Journal of Bifurcation and Chaos, 11(6), 2001.

11. Robert F. Port and Timothy van Gelder, editors. Mind as Motion: Explorations in the Dynamics of Cognition. The MIT Press, Cambridge, MA, 1995.

12. Christine A. Skarda and Walter J. Freeman. How brains make chaos in order to make sense of the world. Behavioral and Brain Sciences, 10:161-195, 1987.

13. Esther Thelen and Linda B. Smith. A Dynamic Systems Approach to the Development of Cognition and Action. The MIT Press, Cambridge, MA, 1994.

14. J. J. Gibson. The Ecological Approach to Visual Perception. Houghton Mifflin, 1979.

15. Esther Thelen. Time-scale dynamics and the development of an embodied cognition. In Port and van Gelder [1], chapter 3, pages 69-100.

16. Rodney A. Brooks. Elephants don't play chess. Robotics and Autonomous Systems, 6:3-15, 1990 .

17. Rodney A. Brooks. A robot that walks: Emergent behaviors from a carefully evolved network. In Randall Beer, R. Ritzmann, and T. McKenna, editors, Biological Neural Networks in Invertebrate Neuroethology and Robotics. Academic Press, 1993.

18. Rolf Pfeifer and C. Scheier. Understanding Intelligence. The MIT Press, Cambridge, MA, 1998.

19. Paul F. M. J. Verschure, B. Kröse, and Rolf Pfeifer. Distributed adaptive control: The self-organization of behavior. Robotics and Autonomous Systems, 9:181-196, 1992.

20. Paul F. M. J. Verschure, J. Wray, Olaf Sporns, Giulio Tononi, and Gerald M. Edelman. Multilevel analysis of classical conditioning in a behaving real world artifact. Robotics and Autonomous Systems, 16:247-265, 1995.

21. Paul F. M. J. Verschure. Distributed adaptive control: Explorations in robotics and the biology of learning. Informatik/Informatique, 1:25-29, 1998.

22. I. P. Pavlov. Conditioned Reflexes. Oxford University Press Press, Oxford, 1927.

23. David Kirsh and Paul Maglio. Reaction and reflection in tetris. In J. Hendler, editor, Artificial Intelligence Planning Systems: Proceedings of the First Annual International Conference (AIPS92), Morgan Kaufman, San Mateo, CA, 1992.

24. Rafael Núñez and Walter J. Freeman, editors. Reclaiming Cognition: The Primacy of Action, Intention and Emotion. Imprint Academic, Bowling Green, OH, 1999. 